\title{
L'impact de la dette extérieure publique sur la croissance économique au Maroc : essai d'une modélisation économétrique
}

\author{
TATOUTI Rachid \\ Equipe de Recherches en Management des Organisations sociales et Economie Territoriale \\ Faculté des Sciences Juridiques Economiques et Sociales \\ Université Ibn Zohr - Agadir - Maroc
}

\begin{abstract}
JABHAOUI Seif elislam
Equipe de Recherches en Management des Organisations sociales et Economie Territoriale Faculté des Sciences Juridiques Economiques et Sociales

Université Ibn Zohr - Agadir - Maroc
\end{abstract}

\section{MABROUKI Hassan}

Faculté des Sciences Juridiques Economiques et Sociales

Université Ibn Zohr - Agadir - Maroc

\begin{abstract}
Résumé : La relation entre la dette extérieure publique et la croissance économique dans les pays a toujours fait l'objet de controverses et a écoulé beaucoup d'encre. L'objet de cet article est d'analyser empiriquement l'impact de la dette extérieure publique sur la croissance économique au Maroc durant la période 1983 -2019. Pour ce faire, nous avons utilisé une méthodologie déductive, axée en premier lieu, sur la proposition d'une revue de littérature à la fois théorique et empirique traitant cette problématique. Ensuite, on s'est basé sur une étude économétrique, et précisément une modélisation VAR standard en vue de détecter l'impact du financement externe sur la croissance du PIB au Maroc. A travers l'analyse de fonction de réponse impulsionnelle et de la décomposition de la variance, il s'est avéré que la dette publique n'est pas un déterminant de la croissance économique au Maroc.
\end{abstract}

Mots clés : Dette extérieure publique, croissance économique, VAR.

Digital Object Identifier (DOI): https://doi.org/10.52502/ijesm.v1i2.192 


\section{Introduction}

L'amélioration des conditions de vie de la population et la garantie du bien-être social sont des objectifs cruciaux et substantiels des pouvoirs gouvernementaux de tous les Etats. Or, Quelles que soient les stratégies menées, ces objectifs ne peuvent être réalisés sans une croissance économique durable et inclusive.

La croissance économique est un concept quantitatif, elle est synonyme de production de biens et services et de créations d'emplois et de richesses. Lorsqu'elle est bien exploitée, elle assure une prospérité économique, ainsi qu'une équité sociale et humaine. Elle permettra aux pays de se mettre au diapason du développement. A cet effet, la compréhension de la croissance économique, de ses déterminants et sources a été toujours parmi les préoccupations constantes et un souci impératif des autorités publiques. En effet, plusieurs déterminants ont été soulevés, surtout avec l'émergence de l'école de la croissance endogène, à savoir, le capital humain, l'investissement, l'ouverture commerciale et d'autres, dont on cite par excellence la dette extérieure publique.

L'activité économique d'un pays repose essentiellement sur le bon fonctionnement de ces finances publiques. L'Etat doit combiner entre ses ressources de financement propres et ses besoins de financement en vue de réaliser un solde budgétaire positif. En revanche, dans le cas d'un solde négatif, l'Etat se trouve devant un déséquilibre financier et devant la nécessité du recours au financement interne ou externe. Cette situation de financement via la dette a donné naissance à une panoplie de débats et de controverses sur l'effet de l'endettement public sur l'économie, et en particulier l'effet de l'endettement public externe sur la croissance du PIB. Ainsi, plusieurs études ont été menées en vue de répondre à cette problématique mettant en relation deux variables macroéconomiques.

Au Maroc, l'état des finances devient de plus en plus inquiétant, étant donné que les soldes budgétaires affichaient des chiffres en rouge. Une telle situation caractérisée par des déficits cumulés, a poussé les autorités publiques à s'endetter davantage. En effet, selon le HCP, le taux d'endettement global au Maroc représente $92,5 \%$ du PIB en 2020. Puis, il connaitra malheureusement une hausse de 0,2 point à la fin de l'année 2021, ensuite une brusque montée de 1,2 point en 2022 où il atteindra une proportion de $93,9 \%$ du PIB. Cette tendance haussière de l'endettement sera entretenue par la persistance du niveau élevé du déficit budgétaire. En effet, bien que celui-ci soit en allègement par rapport à son niveau de 2020 où il s'est situé à $7,6 \%$, il demeurera quand même à $6,3 \%$ du PIB en 2021, ce qui devrait entraîner une nouvelle augmentation de la dette publique. A cet égard, cette situation qui semble aggravante, et cette ampleur de l'endettement risque de mettre le pays dans une sphère d'insolvabilité.

Dans cette perspective, ce travail convergera vers l'évaluation quantitative de l'impact de la dette extérieure publique sur la croissance économique au Maroc. Autrement dit, cette étude va essayer de répondre sur la problématique suivante : Quel est l'impact de la dette extérieure sur la croissance économique au Maroc ? Autrement dit, est ce que la dette publique externe est un déterminant de la croissance économique au Royaume.

La démarche adoptée dans ce travail est une démarche déductive, axée sur une méthodologie purement quantitative. En premier lieu, on mettra en place une revue de littérature à la fois théorique et empirique, puis on mettra en exergue une analyse descriptive visant à décrire la structure et l'évolution des variables objet de l'étude. Et finalement, on mettra en œuvre une analyse économétrique qui repose sur une modélisation VAR, en vue d'évaluer l'impact de la dette extérieure publique sur la croissance économique nationale à l'aide du logiciel EVIEWS 12 et sur une période allant de 1983 vers 2019.

\section{Revue de littérature}

Depuis longtemps, plusieurs travaux s'intéressaient à l'étude des différents déterminants et sources de la croissance économique dans les pays. Les résultats affichent que la croissance économique converge vers deux approches, à savoir l'approche exogène et l'approche endogène. Pour la première théorie, en l'occurrence celle exogène, la croissance économique est considérée comme une manne tombante du ciel. Elle est déterminée par des données naturelles (quantité des ressources non renouvelables disponibles), démographiques (croissance de la population et évolution des taux d'activité) et par des évènements non maîtrisables (les progrès technique).

Cependant, une autre réflexion a été développée par la suite mettant en cause l'approche exogène développée par Solow (1956), c'est la théorie de croissance endogène. Cette théorie considère que la croissance est tributaire des comportements des agents et des variables macro-économiques, ainsi, 
qu'au financement international du processus de développement. Elle s'explique par la variation des dépenses engagées dans la production, recherche-développement, des dépenses publiques en formation, aménagements d'infrastructure...etc. Dans les pays émergents, ces dépenses représentent un fardeau pour le budget de l'Etat. Elles sont financées soit par l'épargne nationale soit par l'endettement extérieur de l'Etat.

De ce dernier point, on peut considérer que l'endettement public est une source éventuelle de la croissance économique. A cet égard, plusieurs auteurs se sont penchés sur l'analyse de la relation entre la dette extérieure publique et l'activité économique.

\subsection{Revue de littérature théorique}

L'analyse de la relation qui existe entre l'endettement externe publique et la croissance économique s'avère parmi les champs de recherche fertiles et qui a écoulé beaucoup d'encre. Avec la naissance de la théorie endogène, la croissance économique a été expliquée par plusieurs variables, autres que le capital, le travail et le progrès technique, dont on cite par excellence l'endettement extérieur de l'Etat.

\section{La théorie classique et néoclassique}

La théorie classique considère que l'endettement public est un impôt futur pour l'Etat. En effet, la dette extérieure publique défavorise l'accumulation du capital et la consommation des générations présentes et futures. Pour Adam Smith (1759), l'endettement externe est inefficace et il ne doit pas être utilisé. Selon Adam Smith (1759), L'emprunt extérieur incitera parfois le gouvernement à des dépenses inutiles, ce qui impactera par la suite l'activité économique domestique. Sur la même lignée, Jean Baptiste Say (1799) pense qu'il faut limiter l'emprunt public. Non seulement il alimente la croissance de dépense publique destructrice de la richesse et de la valeur dans l'économie, mais il exige aussi le paiement des intérêts. De ce fait, l'emprunt risque d'avoir un effet boule de neige, qui s'explique par le paiement des intérêts des anciennes dettes peut être effectué avec de nouvelles dettes.

D'après David Ricardo (1817), les agents économiques voient dans l'emprunt une connotation négative. Ils se comportent comme s'ils avaient un impôt ultérieur à rembourser. En effet, ils seront guidés par une anticipation à la hausse des impôts. A cet égard, les ménages augmentent leur épargne, par motif de précaution, au détriment de la consommation pour se protéger contre les hausses des impôts futurs, ce qui aura une influence sur la production, d'où une baisse de la croissance économique.

De même, Barro (1989) a prouvé qu'une politique de déficit budgétaire financé via une source de financement externe reste sans effet sur l'activité économique, dans la mesure où les agents ne sont pas victimes de l'illusion fiscale. En effet, Ces agents anticipent alors une hausse des impôts destinés à rembourser l'emprunt, en constituant une épargne d'un montant équivalent à l'endettement extérieur (Théorème d'équivalence ou théorème de Barro-Ricardo).

En revanche, pour les néoclassiques, trop de dettes causera un ralentissement de l'économie, étant donné que, la plupart des recettes créées seront destinées au paiement de la charge de la dette. En effet, la dette ne sert plus à financer les activités productives, mais plutôt à augmenter le poids de la dette.

Bref, selon Hayek (1989), l'endettement est une croissance économique artificielle. Elle s'appuie sur un niveau d'investissement potentiel supérieur à l'effort de la nation.

En définitive, ces économistes considèrent que toute intervention de l'État est inefficace (principe de l'Etat gendarme). L'État doit assurer tout simplement sa mission régalienne à partir des ressources générées par ses propres sources. Or, d'autres écoles de pensées rejettent ces hypothèses. Elles pensent qu'un déficit budgétaire justifié, maitrisé et financé par des emprunts peut être économiquement efficace.

\section{La théorie du cycle de la dette d'Avramovic (1964)}

Dans cette approche, l'endettement favorise la croissance de la demande. Il entraîne par l'effet accélérateur une augmentation plus que proportionnelle de l'investissement, qui provoque à son tour une hausse de la production. En effet, selon Avramovic (1964), l'amélioration de l'activité économique via l'endettement extérieur s'explique par un cycle composé de trois phases :

$\checkmark$ La première caractérisée par une épargne nationale insuffisante pour financer les investissements, ce qui pousse l'Etat à chercher une source de financement pour pallier à cette insuffisance, à savoir, la dette externe. 
$\checkmark$ La deuxième caractérisée par une augmentation de cette épargne qui permet de financer une part importante des investissements. Mais, elle reste toujours insuffisante, d'autant plus que le poids des intérêts et l'amortissement de la dette se fait sentir. La dette continue donc à croître et ce, jusqu'au seuil culminant où elle cesse d'augmenter.

$\checkmark$ Et en dernière phase, l'épargne intérieure devient supérieure à l'investissement et de l'autre côté on constate une stagnation de la dette extérieure, conjuguée par une évolution des investissements, ainsi qu'une amélioration du niveau de la productivité.

Finalement, cette théorie a été confirmée par les keynésiens, bien qu'elle démontre des insuffisances et des limites. En effet, la majorité des pays émergents semblent piégés dans la première phase du cycle, avec une augmentation excessive de la dette et des performances économiques néfastes.

Finalement, cette théorie présente des limites, vu que la majorité des pays émergents semblent piégés dans la première phase du cycle, avec une augmentation excessive de la dette et des performances économiques médiocres. Mais elle a été confirmée par les keynésiens.

\section{$\checkmark \quad$ La théorie Keynésienne}

Pour les keynésiens, l'endettement ne crée pas des charges ni pour les générations futures ni pour les générations présentes. Les nouveaux investissements créés permettent l'amélioration de l'activité économique domestique, ce qui provoque à son tour un développement économique. Donc, la charge de la dette induite par le financement externe finira par se ralentir. Une telle situation, causera un déficit budgétaire. Mais, selon Keynes, le principe de l'équilibre budgétaire ne doit pas être toujours respecté. En effet, Un déficit budgétaire justifié et maitrisé peut être un facteur de relance économique et de lutte contre le chômage. Encore plus, la stimulation de la demande globale par l'effet multiplicateur est capable de promouvoir une croissance économique durable.

\section{$\checkmark \quad$ La théorie du surendettement}

Les partisans de cette troisième théorie ont essayé de fusionner les deux points précédents, en développant des modèles avec des effets non linéaires de la dette sur la croissance économique Sachs (1988) et Cohen $(1993,1995)$. Autrement, si la dette potentielle du pays dépasse sa capacité de remboursement, les agents économiques anticipent l'augmentation des impôts afin de payer le service de cette dette. Ce qui limite les investissements et c'est la théorie du « surendettement » ou du « fardeau virtuel de la dette » de Krugman (1988).

Cependant la théorie économique avait fini par reconnaître l'existence d'un seuil au-delà duquel, la dette devient insoutenable. Autrement dit, un niveau tolérable et raisonnable de la dette extérieure peut avoir des effets positifs sur la croissance. Mais, un niveau très élevé du stock de la dette impacte négativement la croissance économique.

\subsection{Revue de littérature empirique}

La relation entre la dette publique et la croissance économique d'un pays a toujours fait l'objet de controverses. En effet, plusieurs études se sont engagées pour examiner l'impact de l'endettement public sur la croissance du PIB. Ce point, aura pour objectif de mettre la lumière sur quelques études récentes, qui ont analysé cette relation dans des différents pays.

Shahnawaz Malik et al. (2010), dans leur étude, avaient pour but d'explorer la relation entre la dette extérieure et la croissance économique au Pakistan pour la période 1972-2005, via l'utilisation de la technique économétrique des séries chronologiques. Les résultats de cette étude montrent que la dette extérieure est négativement et significativement liée à la croissance économique.

Encore plus, L'étude de Faraji Kasidi et Makame Said (2013) a examiné l'impact de la dette extérieure sur la croissance économique de la Tanzanie pour la période 1990-2010. Sur la base des données de séries chronologiques sur la dette extérieure et la performance économique, les résultats montrent que la dette extérieure et le service de la dette ont un impact significatif sur la croissance du PIB. L'encours total de la dette extérieure a un effet positif d'environ 0,36939 et le paiement du service de la dette a un effet négatif d'environ 28,517 . Ainsi, Le test de cointégration montre qu'il n'y a pas de corrélation à long terme entre la dette extérieure et le PIB.

De plus, Halima Ibrahim (2013) a cherché d'estimer l'impact de la dette publique externe sur la croissance économique dans quatre pays d'Afrique de l'Est. Il s'agit du Kenya, de la Tanzanie, de 
l'Ouganda et du Rwanda sur une période de 1981 à 2014. Les données ont été analysées en utilisant les techniques d'estimation des modèles à effets fixes et à effets aléatoires. Les résultats ont démontré que la dette externe publique avait un impact négatif sur la croissance économique des pays d'Afrique de l'Est.

Panizza et Presbitero (2014) ont utilisé l'approche de l'instrument variable pour étudier l'impact de la dette publique sur la croissance économique dans les pays de l'OCDE. Leur analyse a révélé une relation négative entre la dette et la croissance économique.

De même, İbrahim Doğan et Faik Bilgili (2014) ont cherché à examiner la relation entre l'endettement extérieur (Privé et public) et les variables de croissance en Turquie sur une période allant de 1974 vers 2009. Cette étude avait comme objectif d'effectuer un duel entre l'endettement public et celui privé pour en chasser le plus favorable pour l'activité économique. En vue de répondre sur cette problématique, le modèle de Markov-switching a été utilisé. L'article indique que, selon les résultats du modèle dynamique multivarié de Markov-switching, premièrement, les emprunts extérieurs publics et/ou privés ont un impact négatif sur la croissance économique. Deuxièmement, l'impact négatif de l'emprunt public sur la croissance économique et le développement excède celui de l'emprunt privé sur la croissance économique et le développement. En définitive, d'après cette étude, l'endettement a des conséquences néfastes sur la croissance quel que soit son type.

L'article de Samir Abdelhafidh (2014), a examiné l'effet de l'endettement extérieur sur la croissance économique en Tunisie. La méthodologie empirique a employé l'approche autorégressive à retards échelonnés et a démontré que la dette externe avait un impact négatif sur la croissance économique sur la période 1970-2010. Ce résultat approuve qu'un allègement et une réduction de la dette externe de la Tunisie aura des répercussions favorables sur la croissance du PIB.

Sur la même route, Leila Ben Ltaief (2014) s'est intéressée à l'étude de l'impact de la dette publique sur la croissance économique. Pour ce faire, un échantillon de 35 pays (zone euro, Union européenne et pays avancés) a été choisi et ensuite divisé en deux catégories selon leurs ratio d'endettement public en pourcentage du PIB. Cette répartition s'est avérée intéressante car elle permettra d'éviter des problèmes d'hétérogénéité. Ainsi, répondre sur cette problématique a exigé l'utilisation d'un outil économétrique se basant sur les estimations de la méthode des moments généralisés en panel dynamique, développée par Blundell et Bond (1998) sur une période 2006-2013. Les résultats obtenus affichent que le groupe des pays qui maitrise bien leurs ratios d'endettement, l'impact de la dette publique est positif. Par contre, l'impact de la dette externe est négatif pour le cas du groupe des pays qui n'arrivent pas à bien maitriser leurs portefeuilles d'endettement. En définitive, cette étude confirme l'apport de la théorie «Debt Overhang » de Krugman (1988), qui stipule que l'endettement est favorable pour la croissance économique, si et seulement si, il est géré avec prudence.

Ainsi, Le travail de Senadza et al. (2017) examine l'effet de la dette extérieure sur la croissance économique en Afrique subsaharienne (ASS). Ces auteurs utilisent des données annuelles pour 39 pays d'ASS de 1990 à 2013 et emploient la technique d'estimation des Méthodes Généralisées des Moments (GMM). Cette étude révèle que la dette extérieure affecte négativement la croissance économique en ASS.

Encore plus, Adelajda Matuka et Shuffield Seyram Asafo (2018) ont examiné examiner l'impact de la dette extérieure sur la croissance économique au Ghana en utilisant des séries chronologiques annuelles de 1970-2017. Les conclusions de cette étude sont que les entrées de dette extérieure stimulent la croissance au Ghana à la fois à long terme et à court terme.

Le travail de Hind Es-Sounboula et Ahmed Hefnaoui (2019) s'intéresse à la problématique de l'impact de la gestion de la dette extérieure sur les agrégats macroéconomiques au Maroc. A cet effet, la technique du modèle VAR structurel a été utilisée en vue de ressortir la nature de relation existent entre la dette extérieure et la croissance du PIB. Les données utilisées couvrent la période de 1998 à 2017. A partir des résultats de l'estimation de notre modèle économétrique, cette étude a révélé que la dette extérieure publique marocaine a un impact positif sur la croissance du PIB.

Une autre étude de Taofik .M. Ibrahim et Abdisamad Mohamed Farah (2020) a examiné la relation entre l'encours de la dette extérieure et la croissance économique en Somalie (1990 à 2016) en utilisant le modèle de correction d'erreur (ECM) en Somalie. Le résultat de l'ECM a montré que le stock de la dette extérieure affecte négativement la croissance économique.

Une autre étude a cherché d'évaluer l'impact de la dette externe sur la croissance économique, à savoir, c'est l'étude de Sima Rani Dey et Mohammad Tareque (2020). Pour ce faire, l'étude évalue la 
cointégration empirique, la dynamique à long terme et à court terme des variables concernées pour la période 1980-2017 au Bangladesh, en appliquant l'approche de test des limites de cointégration ARDL (autoregressive distributed lag). Les résultats de l'étude révèlent l'impact négatif de la dette extérieure sur la croissance du PIB. Sur la même ligne, L'étude d'Abdulkarim Yusuf et Saidatulakmal Mohd (2021) a examiné l'effet de la dette publique sur la croissance économique du Nigeria en utilisant des données annuelles de 1980 à 2018 et la technique de Lag distribué autorégressif. Les résultats empiriques montrent que la dette extérieure constituait un obstacle à la croissance à long terme tandis que son effet à court terme était favorable à la croissance.

La littérature examinée ci-dessus montre qu'une série d'études a été réalisée sur la relation entre la dette publique et la croissance économique. La plupart des études ont tendance à trouver une relation négative entre la dette publique et la croissance économique. En revanche, quelques études ont trouvé une relation non linéaire entre la dette publique et la croissance économique. Cela signifie que la dette publique améliore la croissance économique jusqu'à un seuil de signification au-delà duquel elle affecte négativement la croissance du PIB.

Le débat sur l'effet de la dette publique sur la croissance économique n'est toutefois pas résolu en raison des résultats mitigés des études précédentes. Avant de conclure cette histoire concernant les études empiriques effectuées dans ce champ, on met à votre disposition ci-dessous, un tableau qui présente d'autres études traitant la relation entre les deux variables objet de notre étude et analyse.

Tableau 1: Autres études traitant la relation empirique entre l'endettement public et la croissance économique

\begin{tabular}{|c|c|c|}
\hline Auteurs & Méthodologie adoptée & Résultat de l'étude \\
\hline Boboye et Ojo (2012) & $\begin{array}{l}\text { En utilisant des régressions OLS, Boboye et Ojo } \\
\text { (2012) ont étudié les effets de la dette extérieure } \\
\text { sur la croissance économique au Nigeria. }\end{array}$ & $\begin{array}{l}\text { Ils ont constaté que la dette } \\
\text { extérieure avait un effet négatif sur } \\
\text { le revenu national et le revenu par } \\
\text { habitant du Nigeria. }\end{array}$ \\
\hline Zouhaier et Fatma (2014) & $\begin{array}{l}\text { Zouhaier et Fatma (2014), ont élaboré une étude } \\
\text { sur la croissance économique en relation avec } \\
\text { l'endettement externe public dans } 19 \text { pays en } \\
\text { développement tels que Kenya. }\end{array}$ & $\begin{array}{l}\text { Les auteurs ont constaté que la dette } \\
\text { publique externe en pourcentage du } \\
\text { PIB et du RNB avait un effet négatif } \\
\text { et statistiquement significatif sur la } \\
\text { croissance économique. }\end{array}$ \\
\hline $\begin{array}{c}\text { Onafowora et Owoye } \\
(2017)\end{array}$ & $\begin{array}{l}\text { Cet article utilise un modèle structurel de } \\
\text { croissance économique généralisée à vecteur } \\
\text { autorégressif augmenté d'une variable de dette } \\
\text { pour caractériser l'impact dynamique du ratio de } \\
\text { la dette publique extérieure au PIB sur la } \\
\text { croissance du PIB par habitant au Nigeria sur la } \\
\text { période 1970-2014. }\end{array}$ & $\begin{array}{l}\text { les résultats montrent que les chocs } \\
\text { de la dette extérieure ont des impacts } \\
\text { négatifs de longue durée sur la } \\
\text { croissance économique. }\end{array}$ \\
\hline $\begin{array}{c}\text { Rachid el Bettioui et Aziz } \\
\text { Ouia (2018) }\end{array}$ & $\begin{array}{l}\text { Dans le cadre de ce papier, les auteurs souhaitent } \\
\text { déterminer l'impact de la dette extérieure } \\
\text { publique sur l'activité économique au Maroc } \\
\text { entre } 1994 \text { et } 2013 \text {. Notre méthode repose sur la } \\
\text { modélisation économétrique en utilisant un } \\
\text { modèle linéaire multiple. }\end{array}$ & $\begin{array}{l}\text { Les résultats empiriques ont permis } \\
\text { d'aboutir à la confirmation de la } \\
\text { première hypothèse : la dette } \\
\text { extérieure publique contribue } \\
\text { positivement à l'activité } \\
\text { économique marocaine. Ils } \\
\text { permettent aussi la confirmation de } \\
\text { la deuxième hypothèse : le service } \\
\text { de la dette extérieure publique } \\
\text { influence négativement sur } \\
\text { l'économie marocaine. }\end{array}$ \\
\hline
\end{tabular}




\begin{tabular}{|c|c|c|}
\hline $\begin{array}{c}\text { Inna Shkolnyk et Viktoriia } \\
\text { Koilo (2018) }\end{array}$ & $\begin{array}{l}\text { Cet article examine la relation entre la dette } \\
\text { extérieure et la croissance économique dans les } \\
\text { économies émergentes pour la période 2006- } \\
2016 \text { en Ukraine, via l'utilisation de différents } \\
\text { outils économétriques, par exemple le modèle } \\
\text { ADL et l'analyse de corrélation. }\end{array}$ & $\begin{array}{l}\text { la dette extérieure qui a une } \\
\text { influence de type non linéaire sur la } \\
\text { croissance économique. Autrement } \\
\text { dit, la dette publique aura un impact } \\
\text { positif sur la croissance économique } \\
\text { si elle n'a pas dépassé le seuil de } \\
\text { l'endettement maximal et si elle a } \\
\text { été bien gérée. }\end{array}$ \\
\hline $\begin{array}{l}\text { Oumansour nor-eddine et } \\
\text { chkiriba driss (2019) }\end{array}$ & $\begin{array}{l}\text { Cette étude a pour objectif d'étudier l'impact de la } \\
\text { dette publique externe sur la croissance } \\
\text { économique au Maroc. Les estimations portent } \\
\text { sur la période 1988-2016. L'instrument } \\
\text { économétrique utilisé pour l'estimation des } \\
\text { paramètres du modèle est basé sur la méthode du } \\
\text { "ARDL bound testing". }\end{array}$ & $\begin{array}{l}\text { Les résultats confirment que la dette } \\
\text { publique élevée a un effet négatif et } \\
\text { largement significatif sur la } \\
\text { croissance économique, aussi bien à } \\
\text { court qu'à long terme. L'effet est } \\
\text { beaucoup plus important à court } \\
\text { terme qu'à long terme. }\end{array}$ \\
\hline $\begin{array}{c}\text { Haddou Djamal et } \\
\text { benbayer Habib (2020) }\end{array}$ & $\begin{array}{l}\text { Cette étude cherche à savoir si les responsables } \\
\text { de l'Algérie, du Maroc et de la Tunisie ont alloué } \\
\text { efficacement leurs dettes souveraines pour } \\
\text { améliorer leurs performances économiques au } \\
\text { cours de la période 1970-2016.Pour vérifier cela, } \\
\text { la méthode de régression OLS a été utilisée, en } \\
\text { plus des statistiques de pré-estimation pour } \\
\text { soutenir les résultats du modèle. }\end{array}$ & $\begin{array}{l}\text { Les résultats montrent une mauvaise } \\
\text { allocation des fonds de la dette } \\
\text { souveraine pour améliorer la } \\
\text { performance économique en Algérie } \\
\text { et au Maroc. Ils indiquent également } \\
\text { que les responsables tunisiens ont } \\
\text { réussi à allouer correctement les } \\
\text { fonds de la dette souveraine pour } \\
\text { améliorer leur performance } \\
\text { économique en plus du fardeau qui } \\
\text { se produit en tant que séquelles de la } \\
\text { dette souveraine. }\end{array}$ \\
\hline $\begin{array}{c}\text { Berhanu Getinet et Fikadu } \\
\text { Ersumo (2020) }\end{array}$ & $\begin{array}{l}\text { Cette étude vise à analyser les impacts de la dette } \\
\text { publique externe sur la croissance économique en } \\
\text { Ethiopie par l'approche ARDL en utilisant une } \\
\text { série chronologique de données annuelles de } \\
\text { 1983-2018. }\end{array}$ & $\begin{array}{l}\text { Le résultat de cette étude montre que } \\
\text { l'endettement externe a un effet } \\
\text { négatif sur la croissance } \\
\text { économique en Ethiopie. }\end{array}$ \\
\hline $\begin{array}{c}\text { Msatfa zouheir et essaid } \\
\text { meskini (2021) }\end{array}$ & $\begin{array}{l}\text { Cette étude avait pour objectif d'examiner } \\
\text { l'impact de la dette externe sur la croissance } \\
\text { économique au Maroc entre } 1980 \text { et } 2019 \text { en } \\
\text { utilisant le modèle ARDL (Autoregressive } \\
\text { Distributed Lag model). }\end{array}$ & $\begin{array}{l}\text { Le résultat de cette étude montre que } \\
\text { l'endettement externe a un effet } \\
\text { négatif sur la croissance } \\
\text { économique du Maroc sur le court et } \\
\text { le long terme }\end{array}$ \\
\hline
\end{tabular}

Source : Auteurs

2.3. Développement de l'hypothèse

Notre revue de littérature théorique nous a présenté, en abstract, trois grands volets théoriques :

Tableau 2 : Synthèse de la revue de littérature théorique

\begin{tabular}{|c|c|}
\hline Les théories & $\begin{array}{c}\text { Impact de la dette externe sur la } \\
\text { croissance }\end{array}$ \\
\hline Théorie classique & Impact négatif \\
\hline Théorie d'Avramovic (1964) et la théorie keynesienne & Impact positif \\
\hline La théorie du surendettement & $\begin{array}{c}\text { Impact positif jusqu'à un seuil où } \\
\text { il devient négatif }\end{array}$ \\
\hline
\end{tabular}

Source : Auteurs

De ce fait, et sur la base de ces constats théoriques, notre hypothèse à tester est :

H 1 : l'endettement externe public est un déterminant de la croissance économique au Maroc 
Cette hypothèse sera confirmer ou infirmer par la suite via une application d'une étude quantitative axée sur une approche économétrique.

\section{Dette extérieure publique et croissance économique : Analyse descriptive}

\subsection{Evolution de la dette extérieure publique marocaine}

D'après le bulletin statistique de la dette extérieure publique publié en 2020 par le ministère des finances, l'encours global de la dette s'est établit à 374,3 milliards de dhs contre 339,8 milliards de dhs en 2019, soit une augmentation de 10,1 points. En pourcentage du PIB, l'encours global représentait 34,9\% contre 29,5\% l'année précédente. Cette augmentation constatée de l'encours global de la dette est justifiée par les conséquences néfastes causées par la pandémie COVID-19.

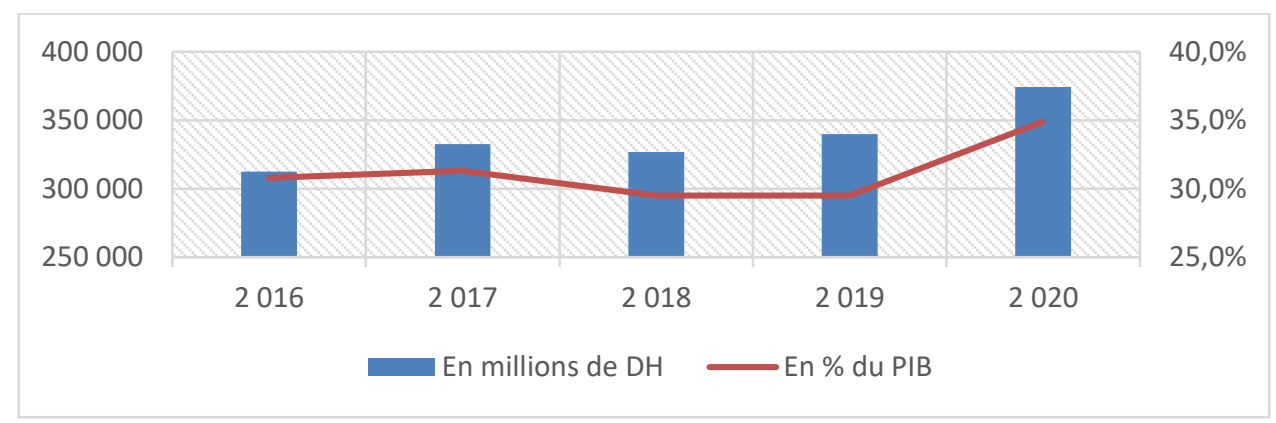

Figure 1 : Evolution de l'encours global de la dette

Pour ce qui est de la structure de l'encours de la dette, selon la répartition par créanciers, les créanciers multilatéraux détiennent $48,9 \%$ de la dette marocaine, soit une baisse de 0,5 points contre l'année antérieure, ensuite les créanciers bilatéraux détiennent $23 \%$ de la dette marocaine contre 25,6\% en 2019 et finalement on trouve le MFI et les banques commerciales avec $28,1 \%$ en 2020 contre $25 \%$ auparavant, soit une hausse de 3,1 point.

Dans une autre vision, cette fois une lecture par répartition des emprunteurs, dans la première place le Trésor rafle plus que la moitié de cette dette avec 53,3\% en 2020, tandis que l'autre part de l'encours est allouée aux établissements et entreprises publics (EEPs) et autres emprunteurs (les collectivités territoriales, les institutions d'utilité publique et les institutions financières publiques). Concernant la structure de l'encours par devises, les statistiques publiées affichent une certaine stabilité en 2020 en comparaison avec les autres années, à l'exception de la part de la dette en dollar qui a connu une légère augmentation passant de $27,1 \%$ en 2019 à $29,8 \%$ en 2020 . S'agissant de la structure de l'encours par taux d'intérêt, la Direction du Trésor des Finances Extérieures (DTFE) indique que 74,5\% de la dette a été accordée à taux fixe, contre $25,5 \%$ à taux variable. Pour ce qui est du service de la dette extérieure, il s'est établi à 41,4 milliards de dhs en 2020 contre 29,2 milliards de dhs en 2019. Ainsi, le tirage sur emprunts extérieurs publics s'est établi à 75,8 milliards de dhs en 2020 , soit une hausse de $92,11 \%$ par rapport à l'année 2019 .

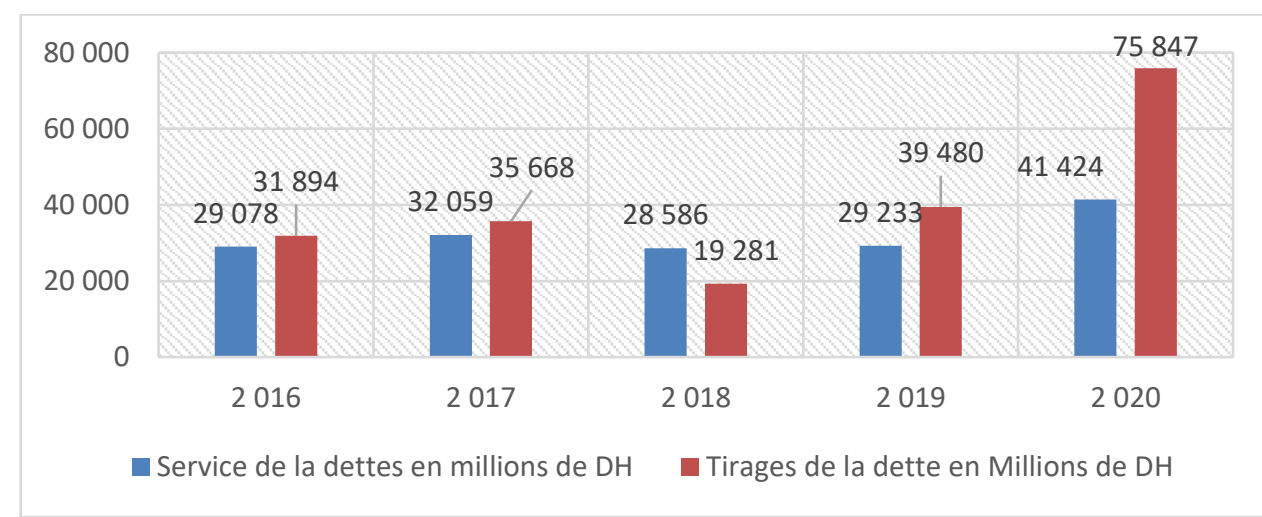

Figure 2 : Evolution du service et du tirage de la dette externe marocaine 


\subsection{Evolution de la croissance économique au Maroc}

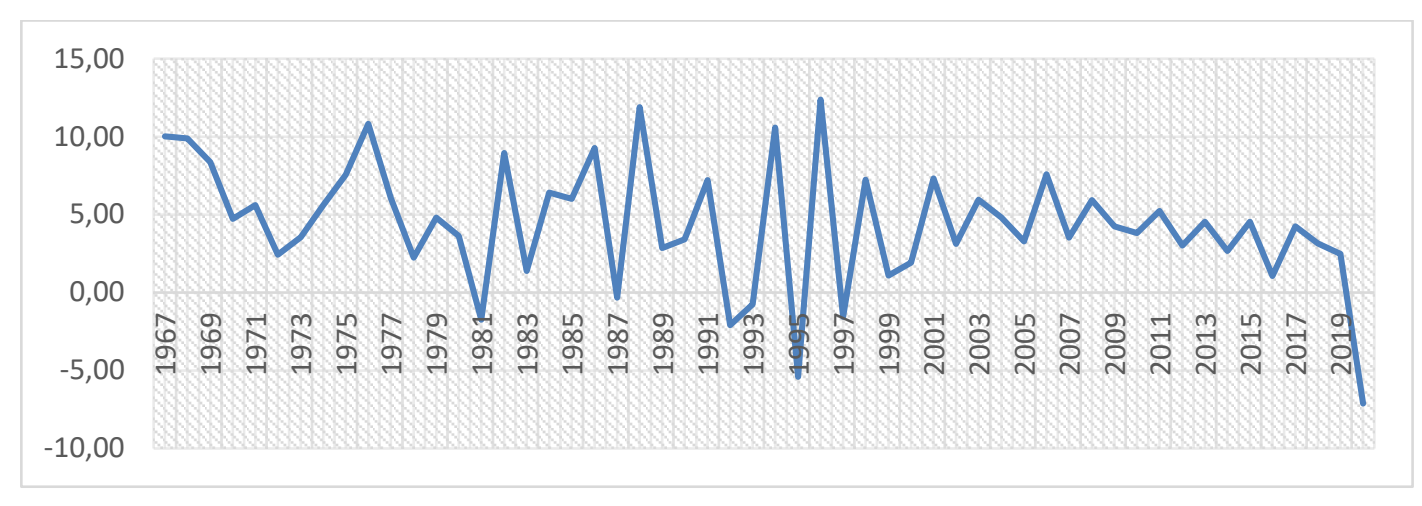

Figure 3 : Evolution de la croissance économique au Maroc

D'après la figure ci-dessus, la croissance économique au Maroc est caractérisée par une évolution en dents de scie, marquant des hauts et des bas selon les variations de la production agricole qui a toujours été fort dépendante des conditions climatiques. En effet, entre 1982 et 2017, la croissance est tombée 5 fois au-dessous de zéro. Cette période avait connu l'enregistrement de la plus grande expansion à long terme (12.37\% en 1996) et aussi d'une contre-performance du taux de croissance en 1995 (- 5.4\%). Certes, l'année 2020 était une année inédite pour tous les pays, et en particulier le Maroc, elle s'est caractérisée par la propagation de la pandémie COVID-19.

En effet, l'économie marocaine avait subi les conséquences néfastes et désastreuses de cette pandémie, connaissant en 2020 sa première récession depuis plus de deux décennies. Le taux de croissance du PIB réel avait enregistré une proportion en noire de 7,12\% contre 2,48\% en 2019. Ainsi, d'après la figure cidessus on constate que les plus importantes performances ont été atteintes lors de la réalisation d'un niveau de croissance décroissant et rapide, pire encore, elles sont souvent suivies par une faible croissance. En conséquence, la croissance économique marocaine connait une forte volatilité qui semble être structurelle et qui gêne le bon fonctionnement de notre économie.

\section{Méthodologie}

L'aspect théorique de ce travail de recherche a été complété par une étude empirique. Celle-ci est menée selon une méthodologie quantitative basée une modélisation économétrique. L'objectif de notre étude est bien évidemment de répondre à la problématique posée, cet objectif réside dans la mise en évidence de l'impact de l'endettement externe public sur la croissance économique au Maroc.

Le choix de la méthodologie quantitative prend son utilité comme le souligne Deslauries (1991) «le principal avantage de l'étude quantitative, par rapport à l'étude qualitative, est qu'elle pouvait fournir des résultats quantifiés pouvant donner lieu à des analyses statistiques. Le second avantage est que l'analyse des données peut permettre les différences entre groupes. Et le troisième avantage est la possibilité d'atteindre en peu de temps un nombre élevé des répondants».

Lorsque nous nous sommes intéressés à l'étude de la relation entre le financement externe et la croissance économique selon une méthode quantitative, notre préoccupation majeure était de détecter est ce que l'endettement public externe est un déterminant de la croissance économique au Maroc. C'est pourquoi, nous avons opté pour une approche économétrique comme outil qui permettra de nous aider à répondre sur notre problématique.

\subsection{Modèle de recherche}

A travers notre revue de littérature, on a conclu que la croissance économique a été expliquée par plusieurs déterminants, dont on cite la dette externe souveraine. Le choix des variables dans cette étude, constituant notre équation, s'est basé sur les travaux de Cecchetti, Mohanty et Zampilli (2011), Checherita-Westphal et Rother (2012) et Minea et Parent (2012).

En définitive, notre modèle prendra la forme simple suivante : 


$$
G D P=\alpha+\beta_{1} E D E P T+\beta_{2} I N V+\beta_{3} I N F L+\beta_{4} P O P+\beta_{5} O U V+\varepsilon
$$

Dont :

- EBEBT : Dette extérieure publique

- INV : Investissement

- INFL : Inflation

- POP : Population

- OUV : Ouverture commerciale

- $\alpha$ : La constante du modèle

- $\varepsilon:$ L'erreur

\subsection{Terrain de l'étude et description}

Cette étude a été effectuée sur le Maroc et à partir des séries temporelles allant de 1983 à 2019. Les données de notre étude sont issues de la banque mondiale (indicateurs de développement mondiaux). A cet égard, et sur la base de notre modèle ci-dessus, on met à votre disposition le tableau suivant qui présente en abstract la description de chaque variable :

Tableau 3 : Description des variables de l'étude

\begin{tabular}{|c|c|c|}
\hline Variables & Description & Mesure \\
\hline $\boldsymbol{G D P}$ & Représente la croissance économique au Maroc. & le taux de croissance du PIB réel. \\
\hline $\boldsymbol{E D E P T}$ & Représente la dette extérieure publique au Maroc. & $\begin{array}{c}\text { le total de la dette externe en \% } \\
\text { du RNB. }\end{array}$ \\
\hline $\boldsymbol{I N V}$ & Représente le taux d'investissement & FBC en \% du PIB \\
\hline $\boldsymbol{I N F \boldsymbol { L }}$ & Représente le taux d'inflation. & $\begin{array}{c}\text { le taux d'inflation (Indice des } \\
\text { prix à la consommation) }\end{array}$ \\
\hline $\boldsymbol{P O P}$ & Représente la croissance de la population. & $\begin{array}{c}\text { Le taux de croissance de la } \\
\text { population }\end{array}$ \\
\hline $\boldsymbol{O U V}$ & Représente l'ouverture commerciale. & Les exportations en \% du PIB \\
\hline
\end{tabular}

Source : Auteurs

\section{Résultats et discussions}

\subsection{Résultats de l'étude économétrique}

5.1.1. Les tests de vérification de la fiabilité des Séries utilisées

Comme il a été déjà déterminé, notre modèle prend la forme suivante :

$$
G D P=\alpha+\beta_{1} E D E P T+\beta_{2} I N V+\beta_{3} I N F L+\beta_{4} P O P+\beta_{5} O U V+\varepsilon
$$

Avant de procéder à l'application de la procédure VAR, des tests de vérification de la fiabilité des séries utilisées s'imposent, à savoir, le test de stationnarité, le test de cointégration et la détermination du retard optimal du modèle VAR à estimer.

$\checkmark \quad$ Etude de la stationnarité des séries

Dans le cadre de l'utilisation des séries temporelles dans une modélisation de type VAR, il est impératif d'étudier leurs stationnarités afin d'éviter les problèmes de régressions fallacieuses. A cet effet, nous avons utilisé le test de la racine unitaire ADF (au seuil de 5\%). L'étude de la stationnarité nous permettra de déterminer l'ordre d'intégration, si la variable est intégrée en niveau c'est-à-dire qu'elle intégrée d'ordre zéro et si la variable admet une stationnarité en différence, c'est-à-dire l'ordre d'intégration peut aller de 1 à n. Les résultats du test de stationnarité sont présentés dans le tableau ci-dessous : 
Tableau 4 : Résultats du test ADF

\begin{tabular}{|c|c|c|c|c|c|c|c|}
\hline \multirow{2}{*}{ Variables } & \multirow{2}{*}{ Modèle } & \multicolumn{2}{|c|}{ En niveau } & \multicolumn{2}{|c|}{ 1 $^{\text {ère }}$ différence } & \multicolumn{2}{|c|}{$2^{\text {ère }}$ différence } \\
\hline & & P.v & O.I & P.v & O.I & P.v & O.I \\
\hline \multirow{3}{*}{$G D P$} & Intercept & 0.000 & $\mathrm{I}(0)$ & 0.013 & $\mathrm{I}(0)$ & 0.001 & $\mathrm{I}(2)$ \\
\hline & Trend and intercept & 0.000 & $\mathrm{I}(0)$ & 0.060 & NS & 0.008 & $\mathrm{I}(2)$ \\
\hline & None & 0.298 & NS & 0.009 & $\mathrm{I}(0)$ & 0.000 & $\mathrm{I}(2)$ \\
\hline \multirow{3}{*}{$E D E B T$} & Intercept & 0.439 & NS & 0.000 & $\mathrm{I}(1)$ & & \\
\hline & Trend and intercept & 0.127 & NS & 0.000 & $\mathrm{I}(1)$ & & \\
\hline & None & 0.172 & NS & 0.000 & $\mathrm{I}(1)$ & & \\
\hline \multirow{3}{*}{$I N V$} & Intercept & 0.318 & NS & 0.000 & $\mathrm{I}(1)$ & & \\
\hline & Trend and intercept & 0.354 & NS & 0.000 & $\mathrm{I}(1)$ & & \\
\hline & None & 0.736 & NS & 0.000 & $\mathrm{I}(1)$ & & \\
\hline \multirow{3}{*}{$I N F L$} & Intercept & 0.007 & $\mathrm{I}(0)$ & & & & \\
\hline & Trend and intercept & 0.014 & $\mathrm{I}(0)$ & & & & \\
\hline & None & 0.000 & $\mathrm{I}(0)$ & & & & \\
\hline \multirow{3}{*}{$P O P$} & Intercept & 0.321 & NS & 0.529 & NS & 0.000 & $\mathrm{I}(2)$ \\
\hline & Trend and intercept & 0.549 & NS & 0.865 & NS & 0.002 & $\mathrm{I}(2)$ \\
\hline & None & 0.359 & NS & 0.113 & NS & 0.000 & $\mathrm{I}(2)$ \\
\hline \multirow{3}{*}{ OUV } & Intercept & 0.859 & NS & 0.000 & $\mathrm{I}(1)$ & & \\
\hline & Trend and intercept & 0.181 & NS & 0.000 & $\mathrm{I}(1)$ & & \\
\hline & None & 0.925 & NS & 0.000 & $\mathrm{I}(1)$ & & \\
\hline
\end{tabular}

Source : Auteurs

D'après ce tableau, on constate que les variables sont stationnaires à des niveaux différents. Autrement dit, la variable «INFL » est intégrée d'ordre zéro, par contre les variables « EDEBT, INV, OUV » sont intégrées d'ordre 1. Enfin, les variables «POP et GDP» sont intégrées d'ordre 2. En définitive, suite aux ordres d'intégrations différents, un modèle VAR standard s'est avéré adéquat.

Test de cointégration

Le test de cointégration de Johannsen est considéré comme un outil d'évaluation et de vérification de l'existence d'une relation de cointégration entre les différentes variables intégrées. A partir des résultats de la stationnarité, nous concluons que les variables ne sont pas stationnaires du même ordre. Ce constat nous a mené à conclure qu'un test de cointégration au sens de Granger n'est pas nécessaire.

La détermination du retard optimal du modèle VAR à estimer

La détermination du retard optimal noté «p » est nécessaire pour effectuer un modèle VAR. pour ce faire, on a fait recours aux critères d'information d'Akaike (AIC) et de Schwarz (SC). Le principe est de retenir le nombre de retards qui minimise les deux critères de choix. Dans notre étude on retient le nombre de retard 4, c'est-à-dire nous allons estimer un modèle VAR d'ordre 4 (VAR(4)).

Tableau 5 : Résultats du retard optimal du modèle VAR à estimer

\begin{tabular}{ccccccc}
\hline \hline Lag & LogL & LR & FPE & AIC & SC & HQ \\
\hline \hline 0 & -371.1025 & NA & 339.5243 & 22.85470 & 23.12679 & 22.94625 \\
1 & -209.7411 & 254.2665 & 0.177110 & 15.25703 & 17.16168 & 15.89789 \\
2 & -146.6021 & 76.53205 & 0.043074 & 13.61225 & 17.14945 & 14.80241 \\
3 & -68.96055 & 65.87771 & 0.006910 & 11.08852 & 16.25827 & 12.82798 \\
4 & 44.60485 & $55.06201^{*}$ & $0.000392^{*}$ & $6.387585^{\star}$ & $13.18989^{*}$ & $8.676354^{*}$ \\
\hline \hline
\end{tabular}

Source : Elaboration à l'aide du logiciel EVIEWS 12 


\subsubsection{Estimation du modèle}

La modélisation vectorielle autorégressive a pour objectif de décrire les interdépendances entre les variables. Les résultats de cette modélisation nous ont permis de déterminer plusieurs relations entre les variables étudiées, en revanche nous nous intéressons à exprimer l'équation de la croissance économique (GDP) en fonction des autres variables pour déceler l'impact de la dette extérieure (EBEBT) sur l'activité nationale. Les résultats se présentent comme suit:

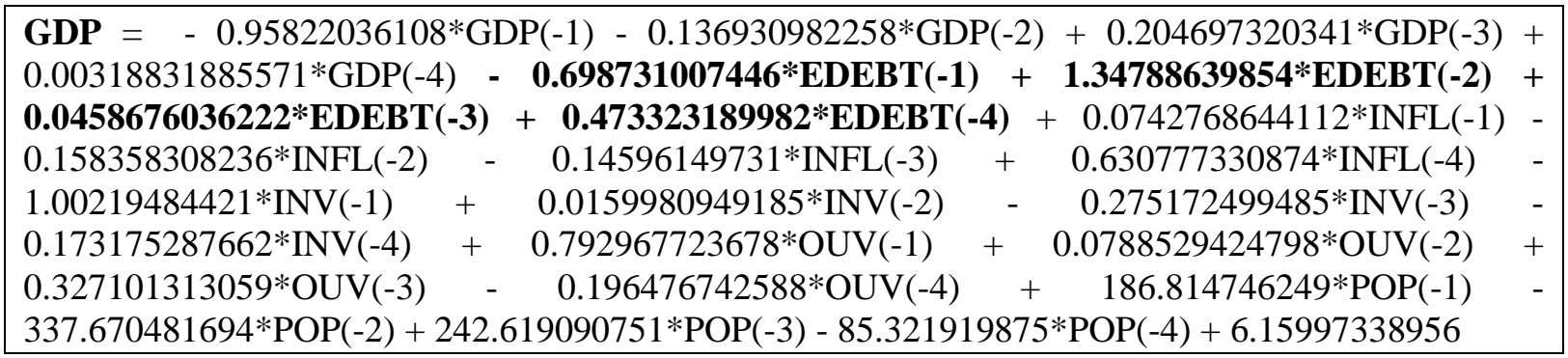

En termes de significativité du modèle, les résultats révèlent un coefficient de détermination $\mathbf{R}^{2}=\mathbf{0 , 8 8}$. Le R-squared est significatif dans la mesure où les variables du modèlent expliquent $88 \%$ la croissance économique.

L'étape suivante consistera à interpréter les résultats issus de notre modèle VAR(4). Or, il convient de tester en premier lieu sa robustesse économétrique.

En effet, une série de tests servent à l'étude de la validité et la robustesse du modèle VAR. Pour ce faire, nous allons appliquer deux tests : il s'agit du test de stationnarité du modèle VAR et du test de normalité de jarque-Bera.

\section{Test de stationnarité du modèle VAR}

Nous allons vérifier le test de stationnarité de notre modèle à partir du test de l'inverse des racines polynômes caractéristiques. Dans ce cas, il faut que l'ensemble des racines soient à l'intérieure du cercle.

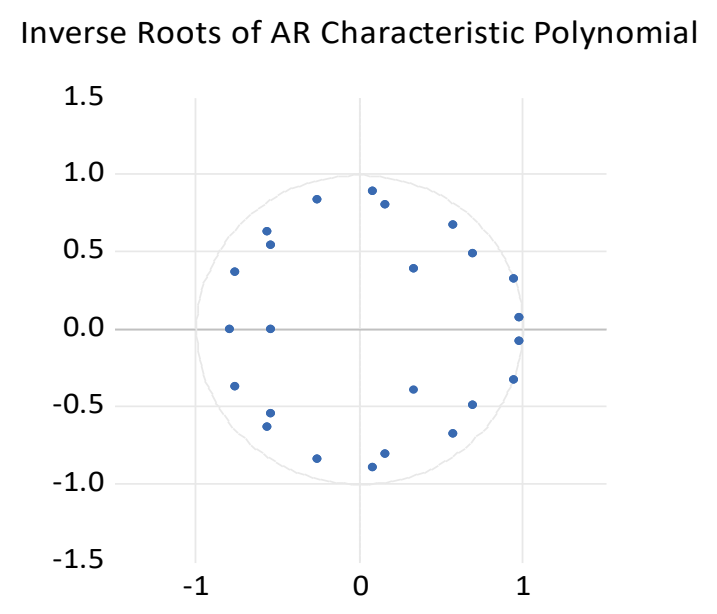

Figure 4 : test de l'inverse des racines polynômes caractéristiques

Nous remarquons que l'intégralité des valeurs propres se situe à l'intérieur du cercle unité. Donc le modèle VAR(4) est stationnaire. Or, on constate que deux points se trouvent sur le cercle. Cette situation est acceptable bien que les valeurs propres de ces deux points dépassent 1 (leurs valeurs est 1,006) mais elles restent proches. 
$\checkmark \quad$ Le test de normalité de jarque-Bera

Le test de normalité de jarque-Bera est un test statistique qui sert à tester si la distribution est normale. Si la probabilité de jarque-Bera est supérieure à 5\%, l'hypothèse de la normalité de la distribution est confirmée. Les résultats de ce test sont présentés comme suit :

Tableau 6 : Le test de normalité de jarque-Bera

\begin{tabular}{cccc} 
Component & Jarque-Bera & $\mathrm{df}$ & Prob. \\
\hline \hline 1 & 1.498611 & 2 & 0.4727 \\
2 & 7.397755 & 2 & 0.0248 \\
3 & 1.194709 & 2 & 0.5503 \\
4 & 4.080455 & 2 & 0.1300 \\
5 & 0.965465 & 2 & 0.6171 \\
6 & 0.387211 & 2 & 0.8240 \\
\hline \hline Joint & 15.52421 & 12 & 0.2140 \\
\hline \hline
\end{tabular}

Source : Elaboration à l'aide du logiciel EVIEWS 12

Les résultats obtenus montrent que la probabilité de notre modèle est égale à 0,2140 . Cette probabilité est supérieure à $5 \%$, ce qui nous laisse dire que les variables de notre modèle suivent une loi normale.

Les tests effectués montrent que notre modèle VAR(4) est stationnaire et que nos variables suivent une loi normale. A cet égard, nous pouvons conclure que notre modèle est valide et prêt à être interprété.

\subsection{Discussions}

\subsubsection{Réponse de Fonction impulsionnelle}

La simulation des chocs structurels constitue une approche puissante dans l'analyse dynamique d'un créneau de variables. Elles reflètent la réaction dans le temps des variables aux chocs contemporains identifiés. Nous allons retracer les réponses aux chocs sur les résidus des variables étudiées et cela sur cinq périodes, considérées comme le délai nécessaire pour que ces dernières retrouvent leur niveau à long terme.

Tous les chocs sont standardisés à $1 \%$ et donc l'axe vertical indique le pourcentage de la variance approximative de la croissance économique en réponse à $1 \%$ de choc sur les autres variables. Les résultats de ce test nous montrent les réactions suivantes (On va s'intéresser à la relation entre GDP et EDEBT) :

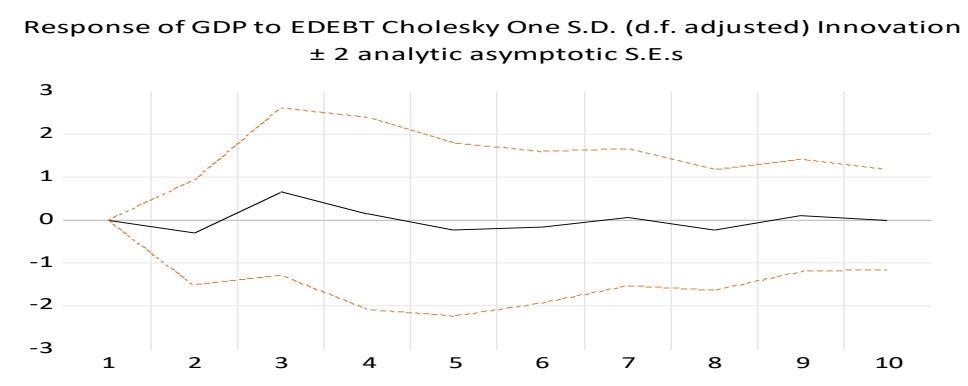

Figure 5 : Réponse de fonction impulsionnelle

Un choc positif de la dette extérieure de $1 \%$ génère un effet négatif de $-0,3 \%$ sur la croissance économique pendant la deuxième période, puis un effet positif de $0,7 \%$ pendant la troisième période, ensuite on constate qu'un choc positif de la dette externe sur la croissance économique est faible. Ce résultat, indique que la dette externe ne contribue pas à l'amélioration de la productivité au Maroc. 


\subsubsection{Décomposition de la variance}

Tableau 7 : Résultats de la décomposition de la variance

\begin{tabular}{|c|c|c|c|c|c|c|c|}
\hline \multicolumn{8}{|c|}{ Variance Decomposition of GDP: } \\
\hline Period & S.E. & GDP & EDEBT & INFL & INV & OUV & POP \\
\hline 1 & 2.603926 & 100.0000 & 0.000000 & 0.000000 & 0.000000 & 0.000000 & 0.000000 \\
\hline 2 & 4.663598 & 86.32080 & 0.356141 & 2.912140 & 6.199982 & 2.719999 & 1.490938 \\
\hline 3 & 5.923560 & 86.42266 & 1.459475 & 1.849500 & 4.959883 & 2.888890 & 2.419591 \\
\hline 4 & 6.541437 & 80.50149 & 1.249252 & 2.302173 & 9.394246 & 3.534396 & 3.018442 \\
\hline 5 & 6.601397 & 79.05118 & 1.332175 & 2.261810 & 9.395278 & 3.799102 & 4.160459 \\
\hline 6 & 6.611495 & 78.81543 & 1.389803 & 2.306725 & 9.385819 & 3.818644 & 4.283580 \\
\hline 7 & 6.911112 & 78.54545 & 1.280986 & 2.293964 & 10.41954 & 3.522596 & 3.937466 \\
\hline 8 & 6.997498 & 76.75071 & 1.357399 & 2.244375 & 11.43541 & 3.704397 & 4.507708 \\
\hline 9 & 7.064660 & 75.69539 & 1.354563 & 3.045019 & 11.69570 & 3.698127 & 4.511201 \\
\hline 10 & 7.093068 & 75.13332 & 1.343851 & 3.139498 & 11.67812 & 3.832249 & 4.872965 \\
\hline
\end{tabular}

Source : Elaboration à l'aide du logiciel EVIEWS 12

Concernant la dette extérieure publique «EDEBT », elle contribue en moyenne de $1 \%$ de la variance de la croissance économique «GDP » entre la troisième année et la dixième année. Ce résultat démontre que le financement externe public ne joue pas un rôle important comme déterminant de la variation de la variable « GDP » au Maroc.

Pour la variable inflation, la contribution est en moyenne ne dépasse pas les $4 \%$ de la variance de GDP. Ce résultat démontre que le Maroc adopte une politique qui converge vers la stabilisation des prix.

Pour la variable investissement, la contribution est de $4 \%$ en moyenne dans la troisième période, puis on constate une augmentation, qui n'est pas forte entre la quatrième période et la dixième période, qui nous mène vers une contribution en moyenne de $11 \%$. En effet, le taux d'investissement au Maroc, malgré les efforts élaborés par les autorités publiques en termes d'attractivité des IDE, demeure très faible. Ce constat explique parfaitement cette contribution faible de l'investissement dans la variance du GDP.

Pour l'ouverture commerciale, la contribution dans la variance du GDP ne dépasse pas le cap de 4\% entre la quatrième période et la dixième période. Ce résultat démontre que l'ouverture commerciale ne peut pas être considérée comme un déterminant important de la croissance économique au Maroc.

Pour le cas du taux de croissance de la population (POP). Conformément aux implications des modèles théoriques et empiriques de croissance économique, une hausse du capital, physique ou humain, entraine une augmentation de la croissance économique. D'après le tableau ci-dessus, la variable « $\mathrm{POP}$ » contribue en moyenne de $4 \%$ entre la cinquième période et la dixième période. Ce résultat indique que le taux de croissance de la population ne détermine pas forcément la croissance économique au Maroc. Finalement, on constate que la variance du GDP a un impact sur elle avec une moyenne de $80 \%$ durant cette période.

On conclut d'après cette analyse via la décomposition de la variance, que la dette externe publique ne détermine pas la croissance économique au Maroc.

En définitive, le graphique suivant nous démontre en abstract la contribution faible des variables explicatives :

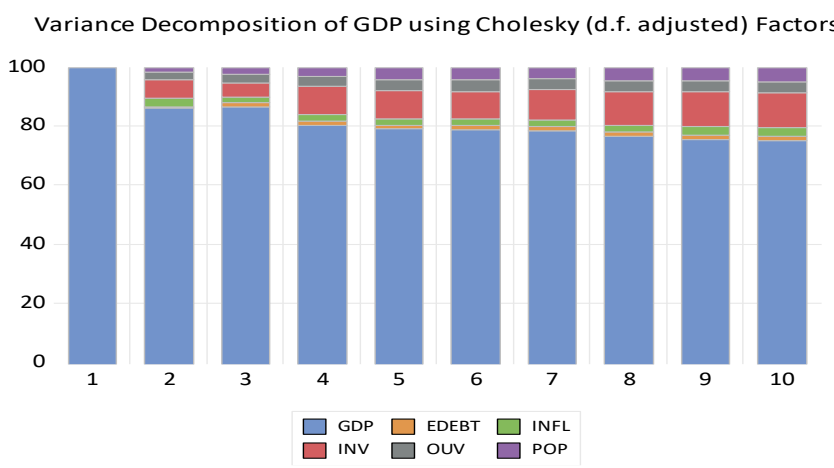

Figure 5 : Représentation graphique de la décomposition de la variance 
D'après notre revue de littérature théorique, on a arrivé à conclure que l'impact de la dette externe publique sur la croissance économique semble mitigé. En effet, la dette publique est corrélée négativement avec la croissance selon les classiques. Par contre, Avramovic (1964) et les keynésiens dévoile l'existence d'une relation positive entre le financement externe et la croissance économique. Et en dernier lieu, on trouve la théorie du surendettement ou du «fardeau virtuel de la dette » de Krugman (1988). Cette pensée théorique arrive à démontrer que l'endettement est favorable pour la croissance économique, mais à condition de ne pas dépasser un seuil de signification donné.

Ainsi, D'après notre revue de littérature empirique, on a capté l'existence aussi d'une série d'études traitant cette relation entre la dette externe publique et la croissance. Il en ressort que l'impact semble différent d'un pays vers un autre, ce qui nous mène vers la constatation d'un bilan empirique mitigé.

Cependant, cette étude menée sur le Maroc a pu déceler un effet néfaste de la dette publique sur la croissance économique sur la base des deux analyses, à savoir, l'analyse de la décomposition de la variance et l'analyse par fonction de réponse impulsionnelle. Ce résultat nous mène vers la non validation de notre hypothèse de recherche et semble contradictoire à la théorie d'Avramovic (1964) et à la théorie Keynesienne. En revanche, notre résultat converge vers la pensée classique qui stipule que l'endettement public n'est pas un catalyseur de la croissance économique.

\section{Conclusion}

L'analyse de l'impact de l'endettement public externe sur la croissance économique par le biais d'une approche économétrique en séries temporelles, nous a permis de déceler beaucoup de conclusions et de remarques qui sont importantes :

Certaines variables du modèle ne sont pas stationnaires. Ceci nous a amené à procéder à leurs stationnarités en recourant à la différenciation. Néanmoins, nous n'avons pas trouvé de relation de cointégration entres les variables vu qu'elles sont intégrées différemment, ce qui fait que la possibilité de construire un modèle de correction d'erreur (VECM) était écartée. Ceci nous a conduits vers l'application d'un modèle VAR standard.

Le test de la détermination du retard optimal associé au modèle VAR nous a permis de retenir un nombre de retard égal à 4.

A partir des résultats de l'estimation de notre modèle économétrique, nous remarquons que les résultats sont conformés à la réalité : la dette extérieure publique marocaine n'a pas d'impact significatif sur la croissance économique. Le présent résultat confirme l'apport de la théorie classique selon laquelle une dette prise par un pays n'aura pas un impact significatif sur la croissance du PIB étant donné que cette théorie considère que l'endettement public est un impôt futur pour l'Etat.

Selon les résultats, il ressort que le niveau d'endettement public au Maroc semble nuisible pour la croissance économique dans le pays et peut être considéré comme un handicap au lieu d'un catalyseur. Cette situation semble inquiétante, vu que l'objectif voulu de l'endettement ne semble pas réalisé, ce qui impactera négativement par la suite sur la situation des finances publiques de l'Etat et nous emmènera vers une situation baptisée « boule de neige ».

\section{BIBLIOGRAPHIE}

(1) Abdulkarim Y.\& Saidatulakmal M.| (2021), The impact of government debt on economic growth in Nigeria, Cogent Economics \& Finance, 9:1, 1946249, DOI : 10.1080/23322039.2021.1946249

(2) Awan A, G \& Humaira Q. (2020). Impact of external debt on ecoomic growth of pakistan. Global Journal of Management, Social Sciences and Humanities, Vol 6 (1), pp.30-61, DOI: https://orcid.org/0000-0001-5767-6229.

(3) Al-Tamimi, K, A. \& Jaradat, M, S. (2019). Impact of External Debt on Economic Growth in Jordan for the Period (2010-2017), International Journal of Economics and Finance; Vol. 11, No.4, doi:10.5539/ijef.v11n4p114. 
(4) Ben Ltaief, L. (2014). Dette publique et croissance économique : investigation empirique pour la zone euro, l'Union européenne et les pays avancés. L'Actualité économique, 90(2), 79-103. https://doi.org/10.7202/1027973ar.

(5) Bennour Smida I. (2016).Problématique de l'endettement Public : Aperçu théorique et analyse rétrospective de l'endettement public en Tunisie, International Journal of Economics \& Strategic Management of Business Process (ESMB), Vol. 6, pp. 1-6.

(6) Berhanu G. \& Fikadu E. (2020). The Impact of Public External Debt on Economic Growth in Ethiopia: The ARDL Approach to Co-integration, Journal of Economics and Sustainable Development, Vol.11, No.11, DOI: 10.7176/JESD/11-11-03.

(7) Boboye, A, L \& Ojo, O, M (2012). Effect of External Debt on Economic Growth and Development of Nigeria, International Journal of Business and Social Science, Vol. 3 No. 12.

(8) Cecchetti S., Mohanty M S and Fabrizio Z. (2011), The real effects of debt, BIS Working Papers, No 352, ISSN 1020-0959.

(9) Doğan, I. \& Bilgili F. (2014). The non-linear impact of high and growing government external debt on economic growth: A Markov Regime-switching approach, Economic Modelling 39, 213 220, www.elsevier.com/locate/ecmod.

(10) Direction du Trésor et des Finances Extérieures (DTFE), Bulletin statistique de la dette extérieure publique (2020), Ministère de l'économie et des finances, Royaume du Maroc.

(11) El Bettioui R. (2018).L'impact du financement par la dette extérieure publique sur la croissance économique marocaine : investigation quantitative, Finance \& Finance Internationale, $N^{\circ} 10$, http://revues.imist.ma/?journal=FFI.

(12) El-Qasemy, M., \& Omari Alaoui, L. Z. (2021). Les moteurs de l'endettement extérieur public du Maroc : investigation empirique 1998-2019. International Journal of Accounting, Finance, Auditing, Management and Economics, 2(3), 1-22. https://doi.org/10.5281/zenodo.4787451

(13) Elwasila S. E. M, (2018). Effect of External Debt on Economic Growth of Sudan: Empirical Analysis (1969-2015), Journal of Economic Cooperation and Development, Pp. 39-62, https://www.researchgate.net/publication/328074036.

(14) Es-sounboula, H. \& Hefnaoui, A. (2019) «Etat des lieux du secteur énergétique marocain » Revue du contrôle, de la comptabilité et de l'audit « Numéro 9 : Juin 2019 / Volume 4 : numéro 1 »Pp : $289-313$.

(15) Faraji, K. \& A. Makame, S. (2013). Impact of External Debt on Economic Growth: A Case Study of Tanzania, Advances in Management \& Applied Economics, vol. 3, no.4, 59-82.

(16) Gövdeli, T. (2019). External Debt and Economic Growth in Turkey: An Empirical Analysis, Sosyoekonomi, ISSN: 1305-5577,Vol. 27(40), 119-130, (https://orcid.org/0000-0002-6600$\underline{8684}$.

(17) Haddou, D. \& benbayer, H. (2020). The Impact of Sovereign Debt on Economic Growth in North Africa: A Comparative Study among Algeria, Morocco and Tunisia, Strategy and Development Review, Volume:10 / N: 01 (2020), p 190-207.

(18) Hadhek Z. \& Mrad F. (2014), Debt and Economic Growth, International Journal of Economics and Financial Issues, Vol. 4, No. 2, 2014, pp.440-448, ISSN : 2146-4138, www.econjournals.com.

(19) Msatfa Z. \& Essaid M. (2021) «La dette externe et la croissance économique au Maroc : une investigation empirique via ARDL», Revue Internationale des Sciences de Gestion « Volume 4 : Numéro 1» pp : 598-618.

(20) Olugbenga O. \& Oluwole O. (2017). Impact of external debt shocks on economic growth in Nigeria: a SVAR analysis, Econ Change Restruct, DOI 10.1007/s10644-017-9222-5. 
(21) Omrane Belguith S., Chakroun M. and Gabsi. B. (2017). Effets non linéaires de la dette publique sur la croissance économique des pays MENA : évaluation empirique à l'aide d'un modèle PSTR, Revue économique et monétaire, $\mathrm{N}^{\circ} 21, \mathrm{Pp} .7-24$.

(22) Oumansour N-E, chkiriba D. (2019). External public debt and economic growth in Morocco: assessment and impacts. International Journal of Economic Sciences, Vol. VIII(2), pp. 80-95., 10.20472/ES.2019.8.2.006.

(23) Oumou G. (2016). Effets de l'Endettement Public sur la Croissance Economique en présence de non linéarité : Cas des pays de l'Union Economique et Monétaire Ouest Africaine. Economies et finances. Université d'Orléans. Français. NNT : 2016ORLE0502. tel-01477847.

(24) Panizza, U., Presbitero, A.F. (2014), Public debt and economic growth: Is there a causal effect?, Journal of Macroeconomics, doi: http://dx.doi.org/10.1016/j.jmacro.2014.03.009.

(25) Samir A. (2014). Dette extérieure et croissance économique en Tunisie, PANOECONOMICUS, 6, pp. 669-689, DOI: 10.2298/PAN1406669A.

(26) Senadza, B. \& Fiagbe, A. K. \& Quartey, Peter (2018) : The effect of external debt on economic growth in Sub-Saharan Africa, International Journal of Business and Economic Sciences Applied Research (IJBESAR), ISSN 2408-0101, Eastern Macedonia and Thrace Institute of Technology, Kavala, Vol. 11, Iss. 1, pp. 61-69, http://dx.doi.org/10.25103/ijbesar.111.07.

(27) Shkolnyk, I. \& Koilo V. (2018). The relationship between external debt and economic growth: empirical evidence from Ukraine and other emerging economies, Investment Management and Financial Innovations, 15(1), 387-400. doi:10.21511/imfi.15(1).2018.32.

(28) Sima R. D. \& Tareque, M. (2020). External debt and growth: role of stable macroeconomic policies, Journal of Economics, Finance and Administrative Science, Vol. 25 No. 50, 2020, pp. 185-204, Emerald Publishing Limited, ISSN 2218-0648, DOI 10.1108/JEFAS-05-2019-0069. 